\title{
Relativistic Jet of Markarian 421: Observational Evidences of Particle Acceleration Mechanisms
}

\author{
Bidzina Kapanadze ${ }^{* a b}$, Stefano Vercellone ${ }^{b}$, Patrizia Romano ${ }^{b}$ \\ ${ }^{a}$ E. Kharadze Abastumani Astrophysical Observatory, Ilia State University, Colokashvili Av. 3/5, \\ Tbilisi, Georgia, 0162 \\ ${ }^{b}$ INAF, Osservatorio Astronomico di Brera, Via E. Bianchi 46, 23807 Merate, Italy. \\ E-mail: bidzina_kapanadzedilauni.edu.ge: \\ patrizia.romano@brera.inaf.it stefano.vercellonedbrera.inaf.it
}

\begin{abstract}
Markarian 421 is one the most extreme blazars characterized by a complex, unpredictable timing/spectral variability, strong X-ray outbursts in some epochs, very broad, nonthermal spectral energy distribution (SED) extending over 16-18 orders of frequency and showing a typical two"hump" structure. The lower-energy component, ranging from the radio to X-rays, is widely accepted to be synchrotron radiation emitted by ultra-relativistic electrons, while the origin of the higher-energy emission (the $\mathrm{MeV}-\mathrm{TeV}$ range) still remains controversial (synchrotron selfCompton, external Compton, hadronic etc.). All these radiative mechanisms need the presence of highly-relativistic particles in the jet, to be initially accelerated via the Blandford-Znajek mechanism and magneto-hydrodynamic processes in the vicinity of the central super-massive black hole. However, particles lose the energy, required for the emission of the $\mathrm{KeV}$ photons, very quickly and the source can maintain its flaring state on the daily-weekly timescales if some additional acceleration mechanisms are continuously at work in the jet. According to the different studies and simulations, particles can gain a tremendous energy due to the propagation of relativistic shocks through the jet: by means of first-order Fermi mechanism at the shock front, or by an efficient stochastic (second-order Fermi) acceleration close to the shock, in the turbulent jet medium. Our intensive X-ray spectral study of Mrk 421 has revealed the dominance of these processes in different epochs: in some periods, the spectral curvature and the photon index showed a positive cross-correlation (expected in the framework of energy-dependent acceleration probability scenario: a particular case of first-order Fermi mechanism), and the source generally demonstrated the a spectral behaviour compatible with the stochastic (second-order Fermi) acceleration (low spectral curvature; anti-correlation between the spectral curvature and the position of the synchrotron SED peak). For our target, relativistic magnetic reconnection seems to be more important during the radio-optical flares. A jet-star interaction process could be the case during 2016 April-August when the source showed a soft gamma-ray excess.
\end{abstract}

High Energy Phenomena in Relativistic Outflows VII - HEPRO VII

9-12 July 2019

Facultat de Física, Universitat de Barcelona, Spain

${ }^{*}$ Speaker. 


\section{Introduction}

Active galactic nuclei represent one of the most interesting and mysterious space phenomena, that produce the bulk of their high-energy (X-ray, gamma-ray) emission in the central compact region whose size can be comparable to that of the Solar system. According to the observational features, active galactic nuclei are divided into different classes and that of BL Lacertae objects (BL Lacs) represent one of the most extreme sites in the universe. Namely, they show a plasma bulk ejection with an apparent superluminal speed due to the movement with a relativistic speed along our line-of-sight. According to the widely-accepted scenario, a super-massive black hole (SMBH, of hundreds millions to a few billion Solar masses) resides in the center of each BL Lac object [1]. Owing to the tremendous gravity, the SMBH is "tugging" the adjacent galactic masses (stars and interstellar matter), which are spiraling towards the event horizon and form a vortex-like structure around it (so-called accretion disc, AD), before being "swallowed" by the central object. The energy stored in the rapidly spinning SMBH extracted and channeled into Poynting flux (socalled Blandford-Znajek mechanism [2]). According to the alternative scenario, AD is threaded by a magnetic field, which is torqued by its rotation and, consequently, can produce a relativistic jet [3]. The jet power, originally carried by magnetically-dominated beam, is progressively used to accelerate matter via the conversion from magnetic into kinetic energy, until a substantial equipartition between the magnetic and kinetic energy fluxes is established [4]. One of the BL Lac jets should be nearly pointed to the observer, its non-thermal emission (with no spectral lines) outshine the thermal emission of other components of the active nucleus [1]. Therefore, the spectral lines of BL Lacs are not detected in the various energy ranges.

However, the aforementioned model of the BL Lac physical nature still is hypothetic, since the active nucleus in encompassed in a very tiny area, and it is impossible to resolve the innermost structure on sub-parsec spatial scales with modern astronomical instruments. Although Markarian 421 (Mrk 421, located at $\alpha=11^{\mathrm{h}} 04^{\mathrm{m}} 25.6^{\mathrm{s}}, \delta=+38^{\circ} 12^{\prime} 4^{\prime \prime}$ ) is one the most nearby BL Lac objects with the redshift $z=0.03$, it is still impossible to resolve the vicinity of the central black hole (see [5] for the observations with the highest spatial resolution reported for this source to date) and study the extreme processes, operating in this region. Therefore, we have to resort to some indirect methods for drawing conclusions about the innermost BL Lac regions.

Some important "landmarks" of the multiwavelength (MWL) study of Mrk 421:

- The source originally was detected as an elliptical galaxy with the UV continuum [6].

- The radio counterpart was detected within Second Bologna Survey of radio sources at $408 \mathrm{GHz}$, denoting it as B2 1101+38 [7].

- Faint absorption lines in the host's optical spectrum were detected and the redshift was determined; a nonthermal nature of the compact central region (featureless spectrum and polarized optical emission), typical for the BL Lac sources, was noticed [8].

- Miller [9] detected a large-amplitude, rapid optical variability with a total range of $\gtrsim 4.7 \mathrm{mag}$.

- Mrk 421 was the first BL Lac source detected in X-rays with the satellite Ariel V [10].

- 1991 May 7-10: the source was detected by the EGRET instrument onboard Compton Gamma-Ray Observatory at $E>100 \mathrm{MeV}$ [11].

- 1992 March-June: The Whipple 10-m Cherenkov-type telescope detected Mrk 421 in the VHE gamma-rays above $500 \mathrm{GeV}$ [12] and this was the first extragalactic detection in this energy range, which was followed by numerous MWL campaigns. 
- Mrk 421 underwent an extreme TeV flare with flux-doubling time of 15 minutes [13].

- Buckley et al. [14] reported the first evidence of a correlated X-ray/TeV variability among BL Lacs, supporting the one-zone synchrotron self-Compton model.

- 2013 April 10-17: unprecedented strong X-ray outburst recorded with Swift-XRT, NuSTAR and INTEGRAL ([15]-[16]).

Similar to other BL Lacs, the broadband spectral energy distribution (SED) of Mrk 421 shows the presence of two hump-like components which are spread from the radio to X-ray frequencies and in the MeV-TeV energy ranges, respectively (see, e.g., [15]). According to the widelyaccepted scenario, the lower-energy one is related to the synchrotron emission from the jet ultrarelativistic electrons (based on the observed radio-optical polarization properties of BL Lacs), while the higher-energy component possibly is generated via the inverse Compton scattering of the lowenergy photons by the ultra-relativistic electrons or via hadronic processes [17]. For the generation of X-ray emission via the synchrotron mechanism or via the up-scatter of the low-energy photons to the $\mathrm{GeV}$ and $\mathrm{TeV}$ energies, it is necessary the presence of an electron population with extreme energies (TeV and higher). However, there are different observational features demonstrating the need of the particle re-acceleration inside the jet over relatively large distances from the central engine after the initial collimation (via the Blandford-Znajek or the magneto-hydrodynamical processes in the inner accretion disc; see below).

Due to the proximity and high X-ray brightness, Mr 421 provides us with an excellent space laboratory for testing different hypothetic mechanisms, responsible for the particle acceleration up to ultra-relativistic energies inside the jet. In this paper, we discuss these processes and summarize their viability via our intensive spectral and cross-correlation study.

\section{Plausible Particle Acceleration Processes}

Similar to other BL Lacs, Mrk 421 shows some observational features, which are explained via the presence of different instable processes inside the jet to be responsible for the particle acceleration to extremely high energies. Namely,

- Electrons should lose the energies, required for the production of X-ray photons very quickly (during a few hours for the magnetic fields inferred from the broadband SED modelling of BL Lacs; see [15]) at the distances of $\sim 100$ Schwarzschild radii. Therefore, the high keV$\mathrm{GeV}$ states, observed on daily-weekly and, sometimes, on monthly timescales, as well as the origin of X-ray emission at significantly larger distances from SMBH (to avoid the annihilation of the $\mathrm{TeV}$ photons via the pair production and a correlated X-ray-TeV variability in Mrk 421, to be related to the same emission zone for X-ray and $\mathrm{TeV}$ photons) can not be explained by the systematic acceleration via the Blandford-Znajek and/or Blandford-Payne mechanisms.

- X-ray spectra of Mrk 421 mostly show a good fit with the logparabolic model $F(E)=$ $K\left(E / E_{1}\right)^{-\left(a+b \log \left(E / E_{1}\right)\right)}$ with the reference energy $E_{1}$ fixed to $1 \mathrm{keV} ; a$, the photon index at the energy $E_{1} ; b$, the curvature parameter; $K$, the normalization factor and the position of the synchrotron SED peak calculated as $E_{\mathrm{p}}=10^{(2-a) / 2 b}$ [17]. Such spectra are generated by an electron population with a lognormal energy distribution, which can not be produced during the systematic acceleration at the jet innermost region. 

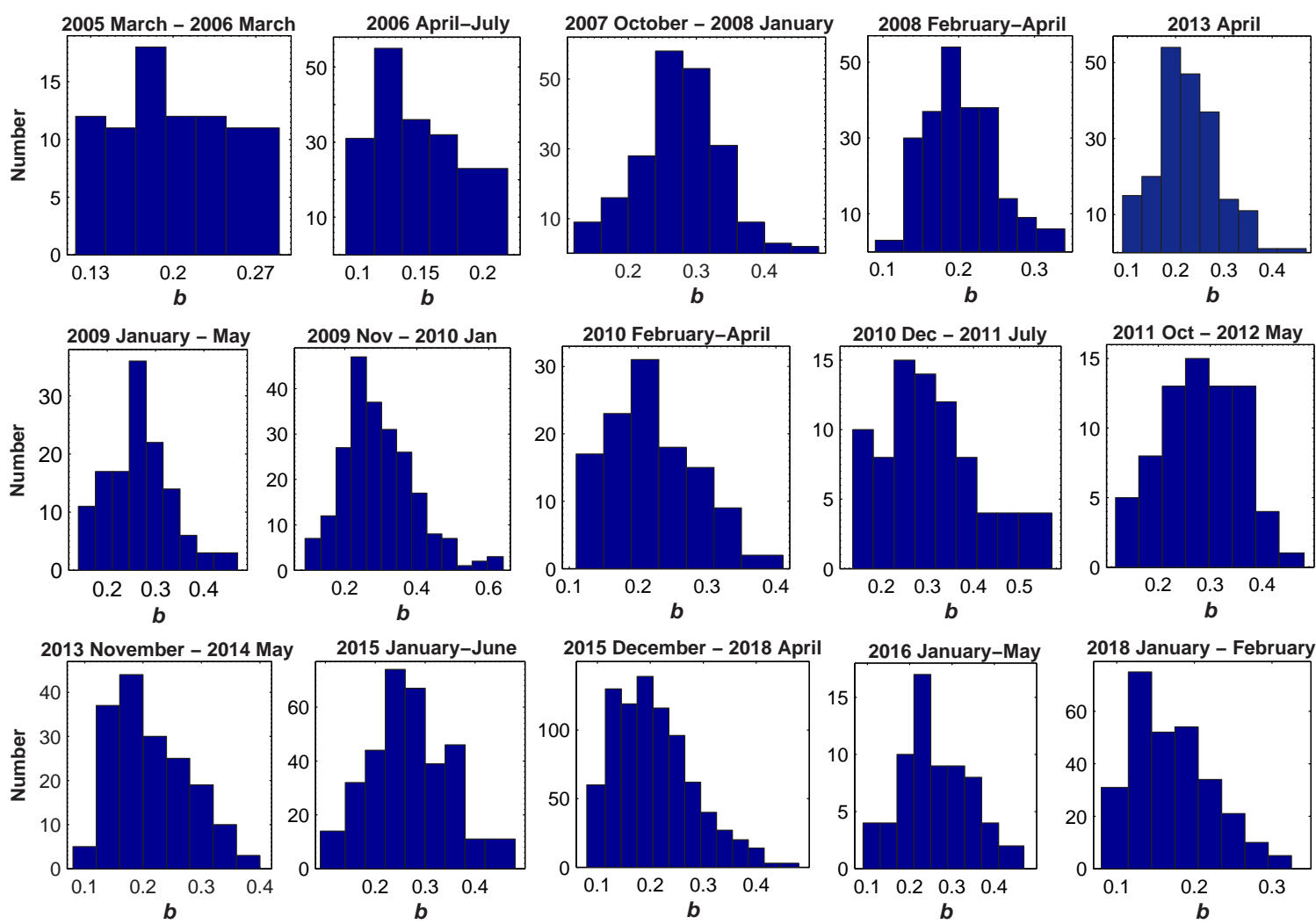

Figure 1: Distribution of parameter $b$ in different periods (adopted from [15], [26]-[29]).

- Rapid TeV and X-ray variability with timescales of a few minutes - shorter, by at least an order of magnitude, than the light-crossing time of the central SMBH with a typical BL Lac mass. Consequently, such extremely variable emission should be produced in small area of the highly relativistic jet rather than in the central region.

The most plausible "in-situ" acceleration mechanisms in the BL Lac jets considered so far are as follows:

- Diffusive shock acceleration (DSA, first-order Fermi mechanism; [19]) at the front of relativistic shocks. A charged particle, when crossing the shock front, is "trapped" between the "mirrors" of magnetic inhomogeneities moving towards each other and the particle will move back and forth between them due to the reflection from the inhomogeneities. Consequently, it may cross the shock front multiple times and gain energy during each crossing. When electrons are confined by the magnetic field with a confinement efficiency that decreases with the increasing gyro-radius (i.e., with energy), the further acceleration probability of the particle will decline with energy, and the electron energy spectrum can be parametrized by a log-parabolic functional shape. The photons emitted by these particles will be distributed as a function of the energy according to the same law (so-called energy dependent acceleration probability scenario, EDAP [18]).

- Stochastic (second-order Fermi) acceleration - developed at the turbulent structures which are generated or strongly enhanced in the shocked jet area [20]. In this case, the scatter centers (e. g., Alfven waves) should move towards each other with some speed $V$ and the particle gains the energy proportional to the square of this speed during each scattering [21]. 

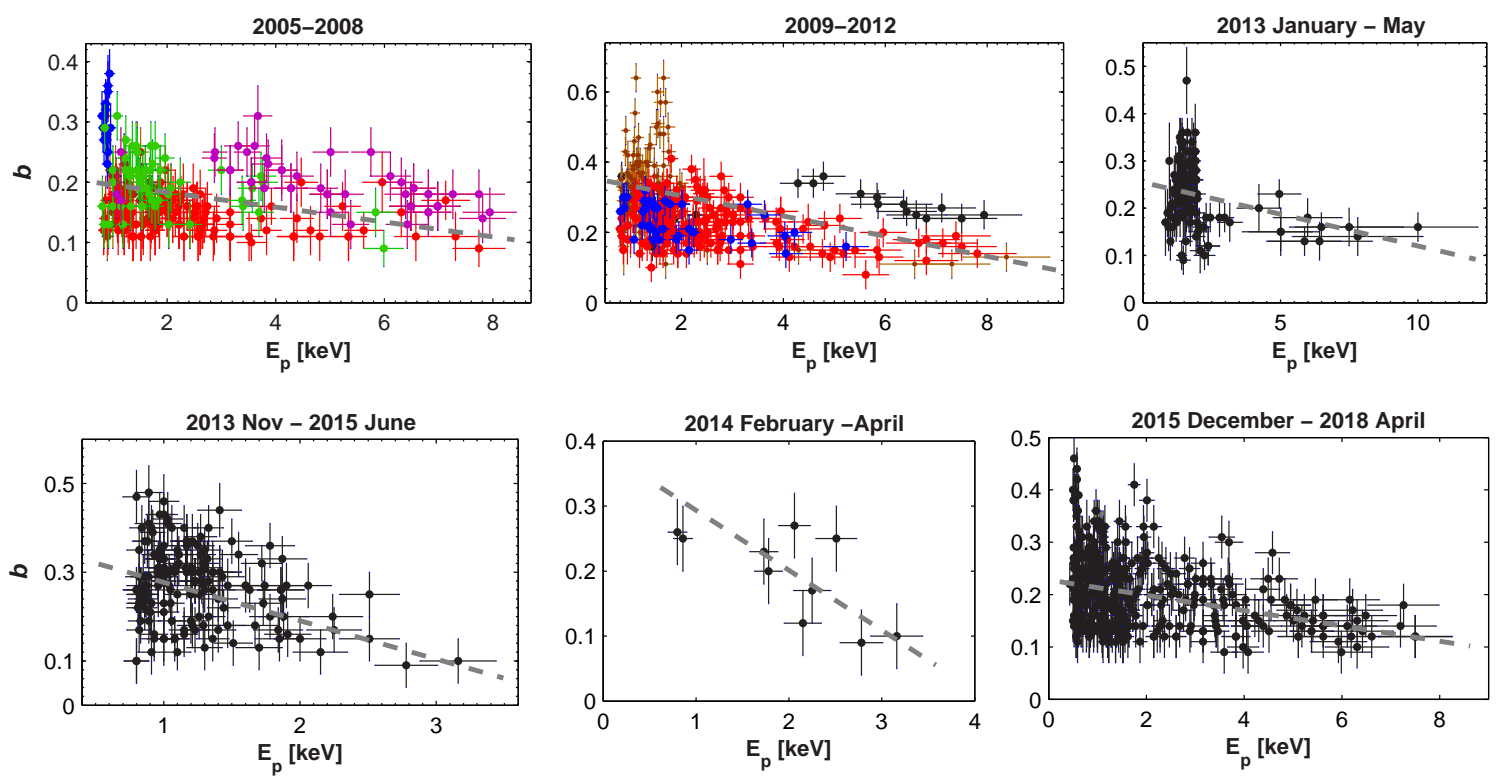

Figure 2: The anti-correlation $E_{\mathrm{p}}-b$ in different periods (adopted from [15], [26]-[29]). Dashed lines represent linear fits to the scatter plots.

The stochastic mechanism does not depend on the bulk velocity of the jet plasma, and the acceleration of charged particles to ultra-relativistic energies even at relatively large distances from the shock front. That is, the stochastic process can accelerate particles during longer times than the first-order Fermi mechanism [22].

- Relativistic magnetic reconnection - driven mostly by kink instabilities, which is an efficient convertor of magnetic energy into bulk motion and particle's energy; expected to operate effectively in highly-magnetized plasmas [23].

- Shear acceleration - a Fermi-type mechanism without shocks, wherever scattering centers flow at different speeds, even if the flows are parallel (e.g., longitudinal shear across the jet radius): particles are intercepted by the difference between the fast core of the jet and the slower exterior [24].

- Jet-star interaction - stellar winds (e.g., bubbles from red giants), interacting with AGN jets, can produce a double bow-shocks structure in which particles can be accelerated to relativistic energies, possibly contributing to the jet's total non-thermal emission [25].

\section{Results}

Our intensive X-ray spectral study ([15], [26]-[29]) has shown that Mrk 421 mostly exhibits a log-parabolic spectral energy distribution, expected in the framework of the efficient first- and second-order Fermi accelerations of electrons (as discussed in the previous section).

According to Massaro et al. [30], the electrons in the jets of the TeV-emitting BL Lacs should undergo a considerably more efficient stochastic acceleration and show wider synchrotron SED (with the curvature parameter $\mathrm{b} \sim 0.3$ or lower) than the objects, not detected at the $\mathrm{TeV}$ frequencies. Moreover, it is expected the presence of the $E_{\mathrm{p}}-b$ anti-correlation in the case of the efficient stochastic correlation [20]. Within our intensive X-ay spectral study, we also have proved the liability of these two hypotheses and, consequently, the importance of the second-order Fermi mechanism is checked. 

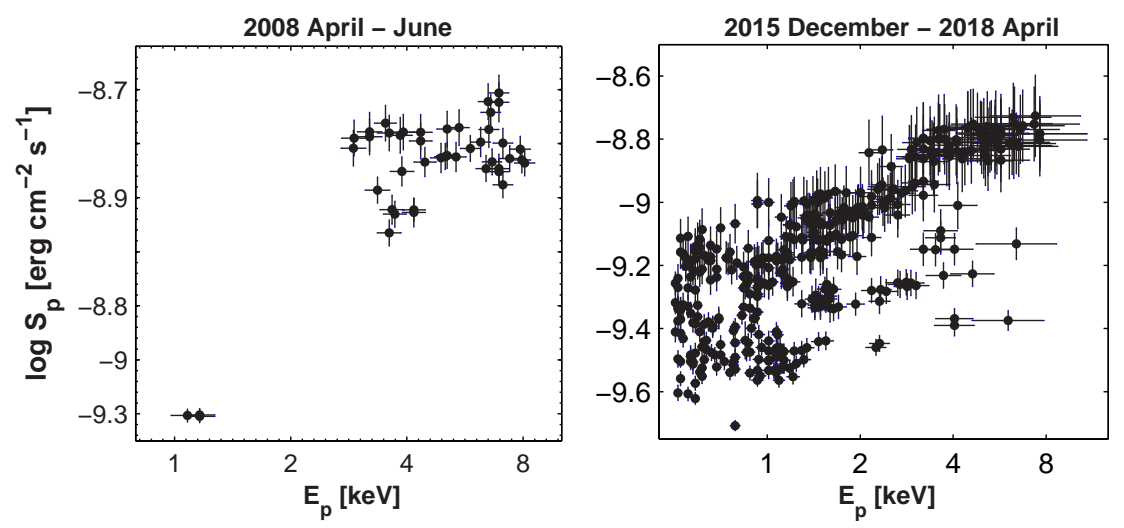

Figure 3: The $S_{\mathrm{p}}-E_{\mathrm{p}}$ relation in different periods (adopted from [27] and [29]).
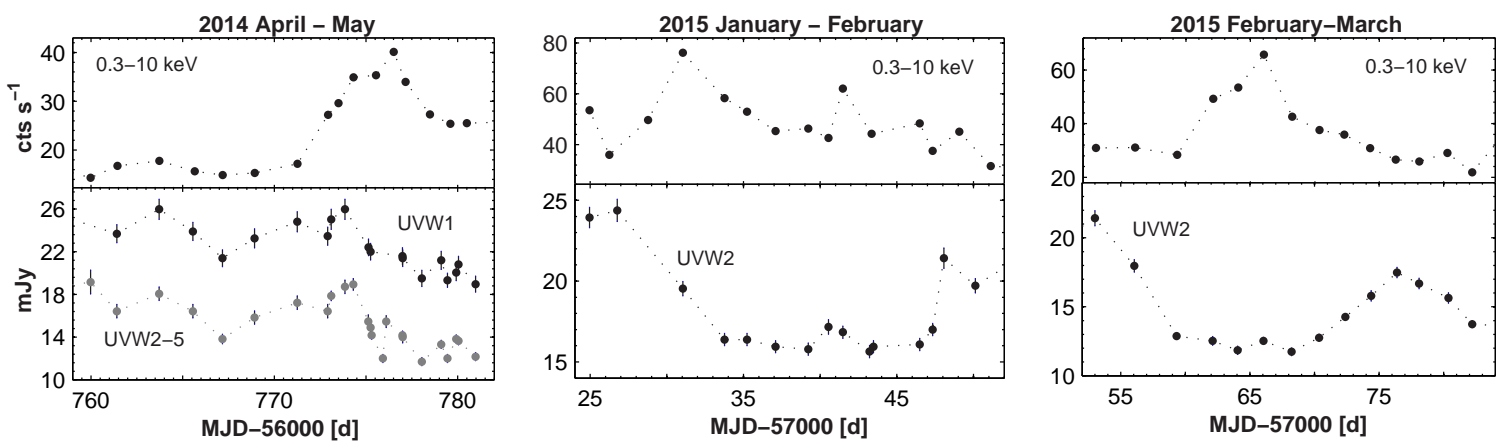

Figure 4: X-ray and UV variability of Mrk 421 in different periods (adopted from [26]).

Figure 1 demonstrates the importance of the stochastic acceleration in the jet of of Mk 421 . Namely, each sub-figure provides the distribution of the values of the curvature parameter $b$ in different periods, obtained from the $0.3-10 \mathrm{keV}$ observations performed with the X-ray Telescope (XRT) onboard the Swift satellite during 2005-2018. They show that Mrk 421 generally is characterized by a wide synchrotron SED with $99 \%$ of the spectra exhibiting b 0.3 or even smaller curvature (i.e. even wider synchrotron SED, inherent to the efficient stochastic acceleration). Our result is in favour of the hypothesis presented by Massaro et al. [30], claiming the efficiency of the stochastic acceleration in the TeV-detected HBL jets.

Moreover, the $E_{\mathrm{p}}-b$ anti-correlation, expected in the case of the efficient stochastic acceleration, generally was detected within our X-ray spectral study of Mrk 421 in the epochs when they showed low spectral curvatures, characteristic for the second-order Fermi process (see Figure 2), although it was mostly weak, hinting at the possibility that the acceleration of X-ray emitting electrons to the ultra-relativistic energies occurred not only via the stochastic mechanism and other physical processes also could be at work. Moreover, this anti-correlation is expected also within EDAP, although this mechanism and stochastic acceleration are expected to produce different slopes in the plane $E_{\mathrm{p}}-b$, which will result in a significant scatter and weakening of the correlation.

We have obtained another interesting result, indicating the importance of the stochastic acceleration in the jet of Mrk 421. Namely, in the case of the log-parabolic spectra, the height of the synchrotron SED peak can be related as $S_{\mathrm{p}} \propto E_{\mathrm{p}}^{\alpha}$ with $\alpha=0.6$ when there is a change in the turbulence spectrum (Figure 3): a transition from the Kraichnan spectrum of the turbulence with 

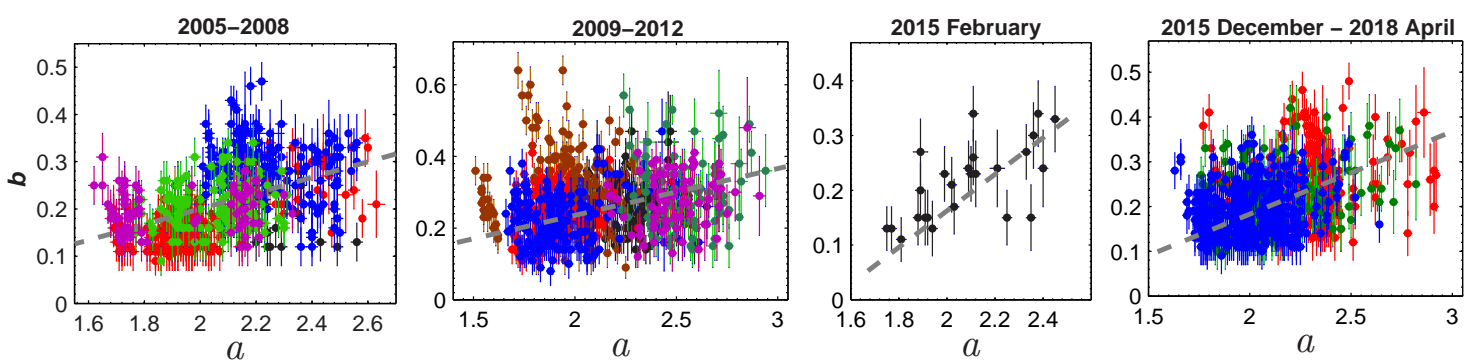

Figure 5: The $a-b$ correlation in different periods (adopted from [26]-[29]). Dashed lines represent linear fits to the scatter plots.

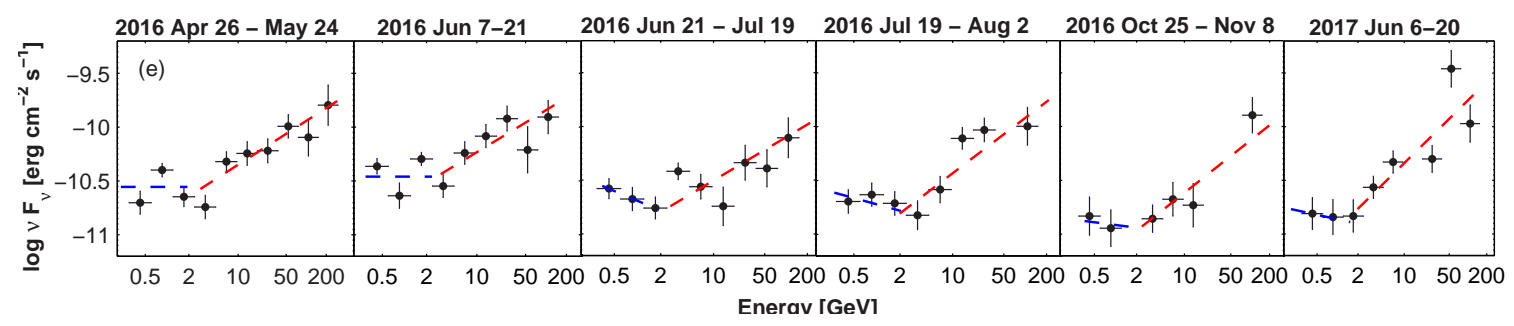

Figure 6: The 0.3-300 GeV SEDs from the time intervals showing a soft gamma-ay excess (adopted from [29]). The blue and red dashed lines represent linear fits to the $0.3-2 \mathrm{GeV}$ and $2-300 \mathrm{GeV}$ SEDs, respectively.

the exponent $Q=3 / 2$ into the "hard sphere"spectrum $(Q=2)$. During the transition, the synchrotron SED follows the expectation of a lower curvature for the harder turbulence spectra [20].

Note that the optical-UV band light curves of Mrk 421 sometimes show a brightness decline when the source had undergone an X-ray flare (see Figure 4). Such anti-correlated variability was explained via the hardening in the electron energy distribution, expected in the case of the stochastic acceleration of electrons with a narrow initial energy distribution, having an average energy significantly higher than the equilibrium energy [31].

The positive $a-b$ correlation, also predicted in the framework of EDAP, was observed in the periods 2005 October - 2006 March, 2006 April-July, 2011 October - 2012 May, 2015 May and 2018 December - 2018 April (see Figure 5). Plausibly, there was a "competition" between EDAP and other acceleration mechanisms not involving this relation ("classical" first-order Fermi process yielding a power-law energy spectrum, stochastic acceleration etc.), resulting in a weakness of the anti-correlation $a-b$, or even its absence in some periods.

Our study revealed a frequent occurrence of soft gamma-ray excess at the energies below $2 \mathrm{GeV}$ during the Fermi-LAT observations of Mrk 421 during 2016 April-November (Figure 6), which could be a contribution from the synchrotron photons produced by ultra-relativistic leptons accelerated in the process of the jet-star interaction [as predicted by Torres-Alba \& Bosch-Ramon [25]).

\section{Summary}

- Mrk 421 shows the need for the particle acceleration processes being at work inside the jet after the initial acceleration and collimation via the Blandford-Znajek and Blandford-Payne mechanisms. 
- Our intensive X-ray spectral study revealed the features predicted by the efficient first and second-order Fermi mechanisms (low spectral curvature, the correlations $a-b$ and $E_{\mathrm{p}}-b$ ) related to the propagation of relativistic shocks and turbulent structures in the jets. The weakness of these correlations hint at the "competition" with other types of acceleration and cooling processes, which are not characterized by such spectral behaviour.

- We observed the correlation $S_{\mathrm{p}} \propto E_{\mathrm{p}}^{0.6}$ in some periods, implying a change in the turbulence spectrum in the jet area producing X-ray emission. The source frequently showed an OpticalUV decline along with X-ray flares, explained by stochastic acceleration of electrons with a narrow initial energy distribution, having the average energy significantly higher than the equilibrium energy.

- We revealed a possible contribution from the jet-star interaction to the to the soft gamma-ray emission in the period 2016 April-August.

\section{Acknowledgements}

BK acknowledge Shota Rustaveli National Science Foundation grant MG-TG-19-360. BK thanks Joseph M. Paredes and LOC for the invitation to the workshop and for the hospitality. PR acknowledges the contract ASI-INAF I/004/11/0.

\section{References}

[1] R. Falomo et al., An optical view of BL Lacertae objects, A\&ARv, 22, 73 (2014)

[2] Blandford, R. D., \& Znajek, R. L., Electromagnetic extraction of energy from Kerr black holes, MNRAS, 179, 433 (1977)

[3] Blandford, R. D., \& Znajek, D. G., Hydromagnetic flows from accretion disks and the production of radio jets, MNRAS, 199, 883 (1982)

[4] A. Tchekhovskoy et al., Efficiency of Magnetic to Kinetic Energy Conversion in a Monopole Magnetosphere, ApJ, 699, 1789 (2009)

[5] B. M. Blasi et al., The TeV blazar Markarian 421 at the highest spatial resolution, A\&A, 559, 75 (2013)

[6] B. Markarian, Galaxies with an ultraviolet continuum, Astrofizika, 3, 55 (1967)

[7] G. Colla et al., The B2 Catalogue of Radio Sources - Third Part, A\&AS, 811, 291 (1973)

[8] M.-H. Ulrich et al., Nonthermal continuum radiation in three elliptical galaxies, ApJ, 198, 261 (1975)

[9] H. R. Miller et al., B2 1101+38: a BL Lacertae object, ApJ, 201, 109 (1975)

[10] M J. Ricketts et al., X-ray transient source at high galactic latitude and suggested extragalactic identification, Nat., 259, 546 (1976)

[111] Y. C. Lin et al., Detection of High-Energy Gamma-Ray Emission from the BL Lacertae Object Markarian 421 by the EGRET Telescope on the Compton Observatory, ApJ, 401, L61 (1992)

[12] M. Punch et al., Detection of TeV photons from the active galaxy Markarian 421, Natur, 358, 477 (1992)

[13] J. A. Gaidos et al., Extremely rapid bursts of TeV photons from the active galaxy Markarian 421, Natur, 383, 319 (1996)

[14] J. H. Buckley et al., Gamma-Ray Variability of the BL Lacertae Object Markarian 421, ApJ, 472, 9 (1996)

[15] B. Kapanadze et al., X-RAY FLARING ACTIVITY OF MRK 421 IN THE FIRST HALF OF 2013, ApJ, 831, 102 (2016) 
[16] E. Pian et al., An active state of the BL Lacertae object Markarian 421 detected by INTEGRAL in April 2013, A\&A, 570, 77 (2014)

[17] H. Krawczynski et al., SIMULTANEOUS X-RAY AND TeV GAMMA-RAY OBSERVATION OF THE TeV BLAZAR MARKARIAN 421 DURING 2000 FEBRUARY AND MAY, ApJ, 559, 187 (2001)

[18] E. Massaro et al., Log-parabolic spectra and particle acceleration in the BL Lac object Mkn 421: Spectral analysis of the complete BeppoSAX wide band X-ray data set, A\&A, 413, 489 (2004)

[19] J. Kirk et al., Particle acceleration and synchrotron emission in blazar jets, A\&A, 333, 452 (1998)

[20] A. Tramacere et al., Swift observations of the very intense flaring activity of Mrk 421 during 2006. I. Phenomenological picture of electron acceleration and predictions for MeV/GeV emission, A\&A, 501, 879 (2009)

[21] G. Cotter, in High-Energy Astrophysics, Oxford University Press (2012)

[22] J. Tammi, \& P. Duffy, Particle-acceleration time-scales in TeV blazar flares, MNRAS, 393, 1063 (2009)

[23] M. Petropoulou et al., Blazar flares powered by plasmoids in relativistic reconnection, MNRAS, 462, 3325 (2016)

[24] F. Rieger, \& P. Duffy, Shear Acceleration in Expanding Flows, ApJ, 833, 34 (2016)

[25] N. Torres-Alba, \& V. Bosch-Ramon, Gamma rays from red giant wind bubbles entering the jets of elliptical host blazars, A\&A, 623, 91 (2016)

[26] B. Kapanadze et al., Mrk 421 after the Giant X-Ray Outburst in 2013, ApJ, 848, 103 (2017)

[27] B. Kapanadze et al., Swift Observations of Mrk 421 in Selected Epochs. I. The Spectral and Flux Variability in 2005Ú2008, ApJ, 854, 66 (2018)

[28] B. Kapanadze et al., Swift Observations of Mrk 421 in Selected Epochs. II. An Extreme Spectral Flux Variability in 2009Ü2012, ApJ, 858, 68 (2018)

[29] B. Kapanadze et al., SWIFT OBSERVATIONS OF MRK 421 IN SELECTED EPOCHS. III. EXTREME X-RAY TIMING/SPECTRAL PROPERTIES AND MULTIWAVELENGTH LOGNORMALITY IN 2015 DECEMBER-2018 APRIL, ApJS, submitted (2019)

[30] F. Massaro et al., X-Ray and TeV Emissions from High-frequency-peaked BL Lac Objects, ApJ, 742, $32(2011)$

[31] K. Katarczynski et al., Stochastic particle acceleration and synchrotron self-Compton radiation in TeV blazars, A\&A, 453, 47 (2006) 